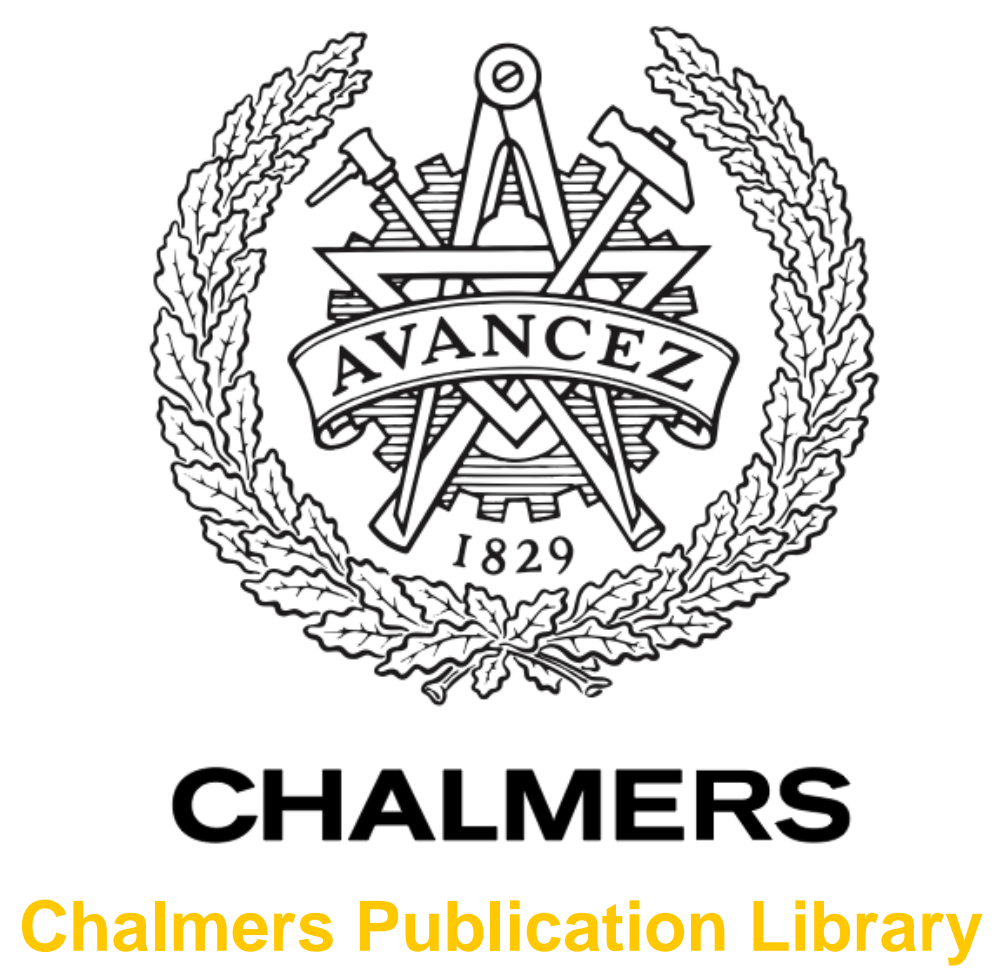

Family of graphene-based superconducting devices

This document has been downloaded from Chalmers Publication Library (CPL). It is the author's version of a work that was accepted for publication in:

JETP Letters (ISSN: 0021-3640)

Citation for the published paper:

Tarasov, M. ; Lindvall, N. ; Kuzmin, L. (2011) "Family of graphene-based superconducting devices". JETP Letters, vol. 94(4), pp. 329-332.

http://dx.doi.org/10.1134/S0021364011160193

Downloaded from: http://publications.lib.chalmers.se/publication/148040

Notice: Changes introduced as a result of publishing processes such as copy-editing and formatting may not be reflected in this document. For a definitive version of this work, please refer to the published source. Please note that access to the published version might require a subscription.

Chalmers Publication Library (CPL) offers the possibility of retrieving research publications produced at Chalmers University of Technology. It covers all types of publications: articles, dissertations, licentiate theses, masters theses, conference papers, reports etc. Since 2006 it is the official tool for Chalmers official publication statistics. To ensure that Chalmers research results are disseminated as widely as possible, an Open Access Policy has been adopted.

The CPL service is administrated and maintained by Chalmers Library. 


\title{
Family of Graphene-Based Superconducting Devices
}

\author{
M. Tarasov ${ }^{a, b}$, N. Lindvall ${ }^{a}$, L. Kuzmin ${ }^{a}$, and A. Yurgens ${ }^{a}$ \\ ${ }^{a}$ Chalmers University of Technology, SE41296 Gothenburg, Sweden \\ e-mail:tarasov@hitech.cplire.ru \\ ${ }^{b}$ Kotel'nikov Institute of Radio Engineering and Electronics, Russian Academy of Sciences, \\ ul. Mokhovaya 11-7, Moscow, 125009 Russia \\ Received July 7, 2011
}

\begin{abstract}
A family of highly sensitive devices based on a graphene nanobridge and superconducting electrodes has been developed, manufactured, and examined. These devices can be used to create a graphene-based integral receiver. A cold-electron bolometer prototype with superconductor-insulator-normal metal tunnel junctions has been studied. Its response to a change in the temperature and external microwave radiation has been measured. A superconducting quantum interferometer with a graphene strip as a weak coupling between superconducting electrodes has been examined. The corresponding modulation of the voltage by a magnetic field at a given current has been measured. The effect of the gate voltage on the resistance of graphene has been analyzed for these samples. To confirm that graphene is single-layer, measurements with the reference samples were performed in high magnetic fields, displaying the half-integer quantum Hall effect.
\end{abstract}

DOI: $10.1134 / \mathrm{S} 0021364011160193$

The attachment of superconducting electrodes to graphene makes it possible to study phenomena involving the proximity effect including multiple Andreev reflection [1,2], as well as to create various devices, such as tunable superconducting chains [3] and superconducting quantum interferometers (SQUIDs) [4]. Nanostructures based on graphene bridges are of interest in view of the creation of highsensitivity receiving and measuring cryogenic instruments, because they have unique characteristics such as the extremely small thickness (one atomic layer), high mobility of carriers, and their zero mass. These parameters make it possible to obtain the extreme characteristics of receiving and measuring instruments that significantly exceed, according to estimates, the existing traditional devices such as bolometers and quantum interferometers. For example, let us estimate the extreme characteristics of a cold-electron bolometer $[5,6]$. Under the action of microwave radiation with power $P$, the electron temperature $T_{e}$ in the millikelvin range is given by the expression

$$
T_{e}^{5}=P(\Lambda \Sigma)^{-1}
$$

where $\Sigma$ is the parameter of the material and $\Lambda$ is the volume of the absorber. To estimate the sensitivity, voltage response $S_{U}$ and the equivalent noise power. The response $S_{U}$ can be written as

$$
S_{U}=\frac{\partial U_{0} / \partial T_{e}}{G_{e-\mathrm{ph}}+G_{\mathrm{SIN}}}
$$

where $G_{e-\mathrm{ph}}=5 \Sigma \Lambda T_{e}^{4}$ characterizes the effective heat transfer between the electron and phonon subsystems of the absorber and $G_{\mathrm{SIN}}=\partial P / \partial T$ is the effective heat transfer of a superconductor-insulator-normal metal (SIN) tunnel junction, which ensures the removal of the hottest electrons from the absorber. Simultaneously, $G_{\mathrm{SIN}}$ ensures electron cooling and makes it possible to avoid the overheating of the absorber. Noise properties are characterized by the equivalent noise power, which can be represented as the sum of three terms in the form

$$
\mathrm{NEP}^{2}=10 k_{\mathrm{B}} \Sigma \Lambda\left(T_{e}^{6}+T_{\mathrm{ph}}^{6}\right)+\delta U_{\mathrm{SIN}}^{2} S_{U}^{-2}+\delta U_{\mathrm{amp}}^{2} S_{U}^{-2} .
$$

Here, the first term is thermodynamic noise of the electron-phonon interaction, the second term is shot noise of SIN tunnel junctions, and the third term describes noise of the following amplifier. According to this formula, a decrease in the volume leads to a strong decrease in equivalent noise power. Under the same other conditions, a change of the standard coldelectron bolometer technology with a 15-nm-thick absorber to 0.3-nm-thick graphene yields a sevenfold gain in equivalent noise power.

Cold-electron bolometers are usually produced by the traditional technology of the deposition of films of aluminum and other metals through a suspended mask at various angles. The lower aluminum layer is oxidized in order to form a tunnel barrier; then, a normalmetal absorber is deposited [7]. In this case, the absorber cannot be thinner than the first layer (50$100 \mathrm{~nm}$ ); for this reason, the volume of the absorber cannot be significantly reduced. We changed the sequence of the deposition of layers; i.e., the absorber is deposited first and its thickness can be reduced to $10 \mathrm{~nm}$ [8]. We previously tried to significantly reduce the volume of the cold-electron bolometer due to the 


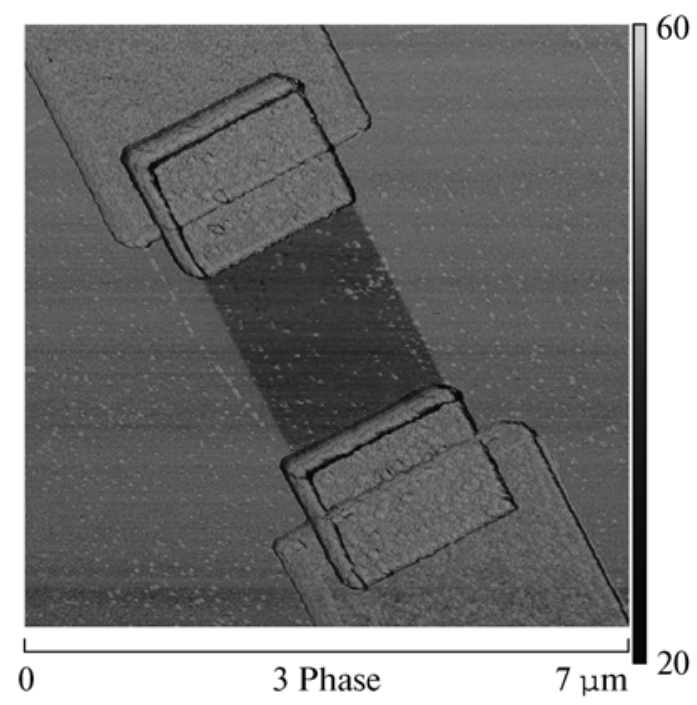

Fig. 1. Atomic force microscope image of the bolometer.

use of carbon nanotubes [9]. These cold-electron bolometers with nanotubes exhibit a finite response to a microwave signal. However, since the contact resistance is too high, the impedances are mismatched and thereby the sensitivity of the device is significantly reduced. The volume of the graphene absorber with an equivalent thickness of $0.3 \mathrm{~nm}$ and the same area is 50 times smaller than the volume of the standard metalfilm absorber with a thickness of $15 \mathrm{~nm}$. In addition, it can be expected that the material parameter $\Sigma$ decreases due to the zero mass of the electrons and holes in graphene.

The cold-electron bolometer consists of an absorber stripe connected to the electrode of an antenna through SIN tunnel junctions on both sides. In our case, the graphene absorber is obtained by the mechanical flaking of graphite flakes and deposition of them on a standard $7 \times 7-\mathrm{mm}$ silicon substrate coated with a silicon oxide layer. Before the deposition of graphene, $\mathrm{Au} / \mathrm{Ti}$ contact sites and film labels for subsequent matching are formed. The coordinates of graphene flakes with respect to labels are determined using an optical microscope. The quantum Hall effect was measured to confirm that the resulting graphene fragments are single-layer. Hall graphene devices manufactured on these substrates exhibit the halfinteger quantum Hall effect characteristic of singlelayer graphene. Flakes in bolometers and interferometers with the same optical contrast are estimated as a single-layer. Using electron lithography, we formed a resist mask through which the plasma etching of graphene in oxygen was performed. As a result, $5.5 \times 2 \mu \mathrm{m}$ graphene bridges are obtained. Then, rectangular $1.5 \times 2 \mu \mathrm{m}$ SIN junctions were manufactured from each edge of the graphene stripe. A junction is a three-layer structure consisting of $1.5 \mathrm{~nm}$ chromium;

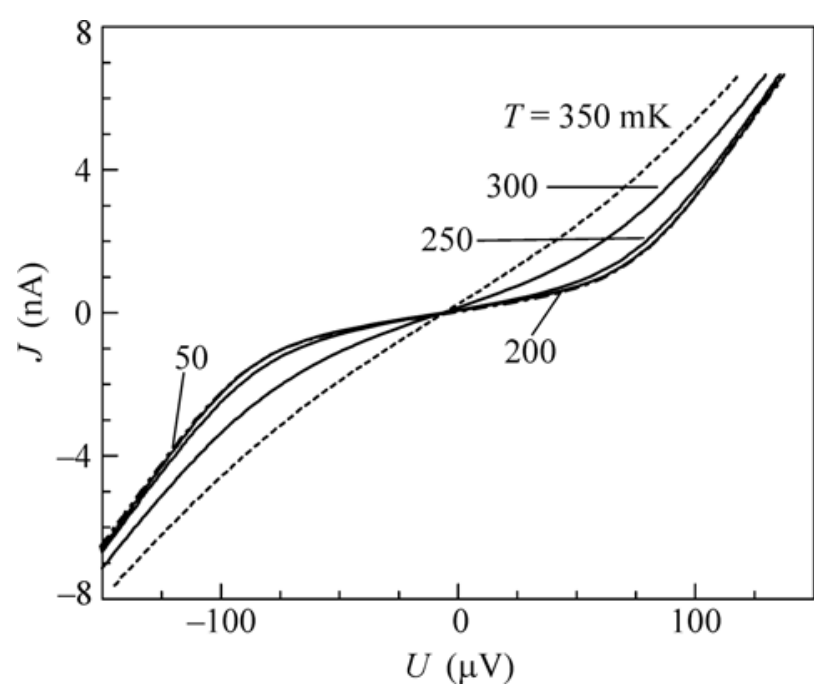

Fig. 2. Current-voltage characteristic of bolometer structures at temperatures of 50,200,250,300, and $350 \mathrm{mK}$.

$10 \mathrm{~nm}$ aluminum and its oxide, which is formed due to oxidation for $5 \mathrm{~min}$ in an oxygen atmosphere at a pressure of $0.05 \mathrm{mbar}$; $70 \mathrm{~nm}$ aluminum; and $5 \mathrm{~nm}$ palladium. After the resist is lifted off in acetone, the third lithography was performed with the formation of 80$\mathrm{nm}$-thick aluminum lead conductors. The first $\mathrm{Cr} / \mathrm{Al}$ layer is not a superconductor. It is a normal electrode of the SIN junction. The atomic-force microscope image of this bolometer with the graphene absorber is shown in Fig. 1. Its current-voltage characteristic and response are plotted in Figs. 2 and 3, respectively.

We also examined the samples with the direct contact of $\mathrm{Al} / \mathrm{Ti}(40 / 2.5 \mathrm{~nm})$ electrodes with graphene without additional SIN junctions. The distance between the superconducting electrodes varied from 0.3 to $3 \mu \mathrm{m}$. The same technology was used to produce SQUIDs. The samples of SQUID constitute an unclosed superconducting loop of an aluminum film with a diameter of $20 \mu \mathrm{m}$ and a graphene bridge in the gap of the loop. The graphene stripe had additional electric contacts through which a current could be fed and the voltage could be taken from the middle of the graphene bridge. Aluminum contacts at the edges of graphene induce a minigap in the normal conductor and ensured the superconducting weal coupling between the ends of the superconducting loop. Samples were manufactured on $7 \times 7$-mm oxidized silicon substrates with 16 contact sites at the edges. Two SQUIDs were formed on one chip. Figure 4 shows the image of one of the SQUIDs. To analyze modulation by the magnetic field, the current can be fed through both the main loop (3/16 contact sites) and an additional loop (6/14 contacts). The current-voltage characteristics of the SQUID were measured by the four-probe method using contacts D1, D2, S1, and $\mathrm{S} 2$. 

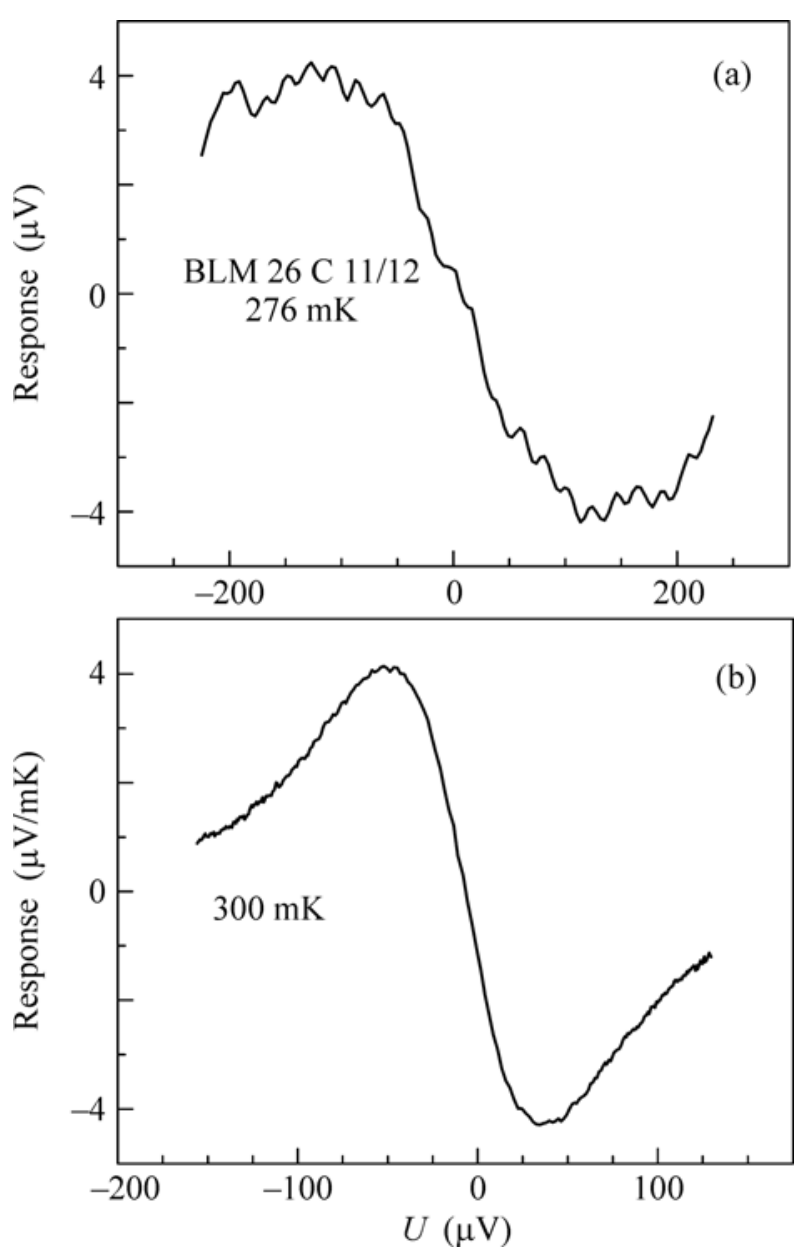

Fig. 3. Voltage response to (a) $110-\mathrm{GHz}$ radiation and (b) temperature change.

The cold-electron bolometers with tunnel junctions, SNS graphene junctions, and interferometers were studied in a TRITON solution cryostat (Oxford Instruments) at temperatures of $50-350 \mathrm{mK}$ and in a HELIOX-ACV sorption cryostat (Oxford Instruments) with an optical window at a temperature of $277 \mathrm{mK}$. To reduce the effect of noise and external magnetic fields, the samples were placed in a double screen consisting of superconducting niobium and cryogenic permalloy. Figure 2 shows the current-voltage characteristic of the sample with SIN junctions at temperatures from 50 to $350 \mathrm{mK}$. It can be seen that the current-voltage characteristic varies slightly below $250 \mathrm{mK}$. An analysis of the effect of $110-\mathrm{GHz}$ radiation on the current-voltage characteristics of these bolometers indicates the voltage dependence of the response that is similar to the corresponding dependences for traditional metal-film bolometers with aluminum electrodes. As is shown in Fig. 3a, the maximum response is observed at bias voltages in the range of $100-150 \mu \mathrm{V}$. The maximum voltage response to a temperature change is $0.4 \mu \mathrm{V} / \mathrm{mK}$ at a bias voltage of $50 \mu \mathrm{V}$, see Fig. $3 b$.

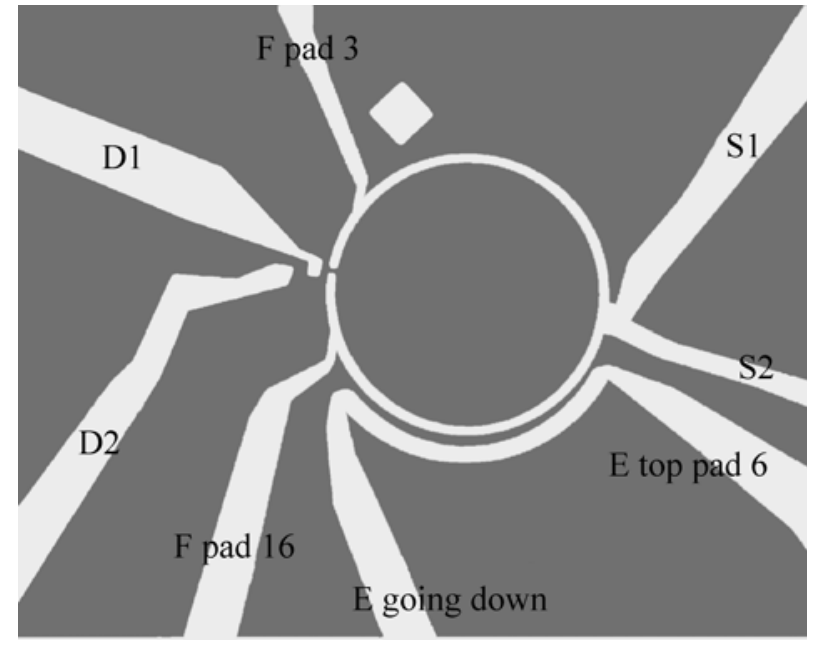

Fig. 4. Structure of the interferometer where contacts D1, D2 and S1, S2 are used for the four-probe measurement of the current-voltage characteristic of the interferometer. The current through the interferometer loop is specified through contacts $F$ pad 3 and $F$ pad 16.

We also examined the current-voltage characteristics and differential resistance of the samples in which aluminum electrodes are in direct contact with graphene. In this case, induced superconductivity appears in graphene and the critical current arises, which can be suppressed by the magnetic field or heating of the sample. Similar effects lead to the appearance of the Josephson weak coupling in the superconducting interferometer. Like in a classical metal-film SQUID, our interferometer exhibits the magnetic field modulation of the critical current. Features of this SQUID are a fairly large resistance $(250 \Omega)$ and hysteresis-free characteristic near $50 \mathrm{mK}$. The magnetic field modulation depth of the voltage at a certain current reached $20 \mu \mathrm{V}$. The current-voltage characteristic of the SQUID remains almost unchanged in the temperature range from 63 to $300 \mathrm{mK}$. The critical current is $0.2 \mu \mathrm{A}$. The normal resistance is $250 \Omega$. The shape of the current-voltage characteristic is somewhat qualitatively different from that for SQUIDs with traditional Josephson junctions and has an excess current. The modulation of the voltage by the current through the induction loop is shown in Fig. 5. The modulation depth is $16 \mu \mathrm{K}$, which is comparable with the value for niobium SQUIDs with bridged SIS junctions corresponds to the voltage-flux sensitivity $d U / d \Phi=50 \mu \mathrm{V} / \Phi_{0}$. A feature of this modulation is its quite extended bias current range up to $4 \mu \mathrm{A}$ or $1 \mathrm{mV}$ (Fig. 6). This is much larger than a critical current of $0.2 \mu \mathrm{A}$. This effect can be due to the process similar to that in an Andreev interferometer, where the resistance is modulated by the magnetic field.

To summarize, a family of superconducting devices with a graphene active element has been developed, manufactured, and examined. Josephson junctions 


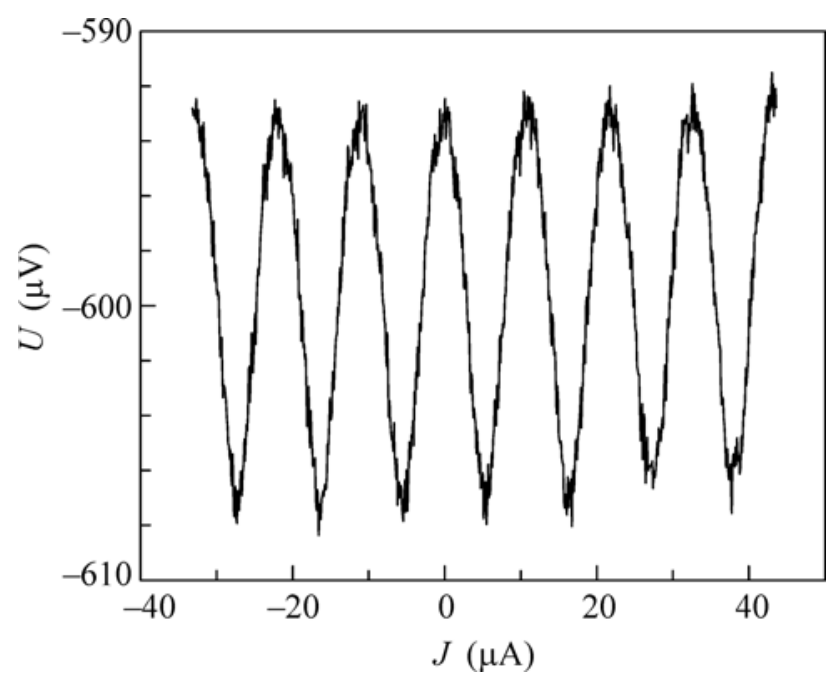

Fig. 5. Modulation of the voltage on the interferometer by the current through the loop of the SQUID.

based on the proximity effect with direct conductivity, as well as superconductor-insulator-normal metal tunnel junctions where graphene is used as the normal metal, have been examined at temperatures in the range of $50-350 \mathrm{mK}$. The voltage response to a temperature change, $d U / d T=0.4 \mu \mathrm{V} / \mathrm{mK}$, and the external microwave radiation $(f=110 \mathrm{GHz})$ have been measured for a cold-electron bolometer prototype. The voltage-flux characteristic of the superconducting quantum interferometer with the Josephson weak coupling with the modulation depth $d U / d \Phi=$ $50 \mu \mathrm{V} / \Phi$ has been measured. The critical current and normal resistance are $0.2 \mu \mathrm{A}$ and $250 \Omega$, respectively. The results and instruments can be used to create a superconducting receiving chip based on graphene with a cold-electron bolometer as a receiver of terahertz radiation and a graphene SQUID as a readout device.

This work was supported by Svenska institutet (Swedish Institute), Vetenskapsrädet (Swedish Research Council), Stiftelsen før strategisk forskning (Swedish Foundation for Strategic Research), and the Russian Foundation for Basic Research, project

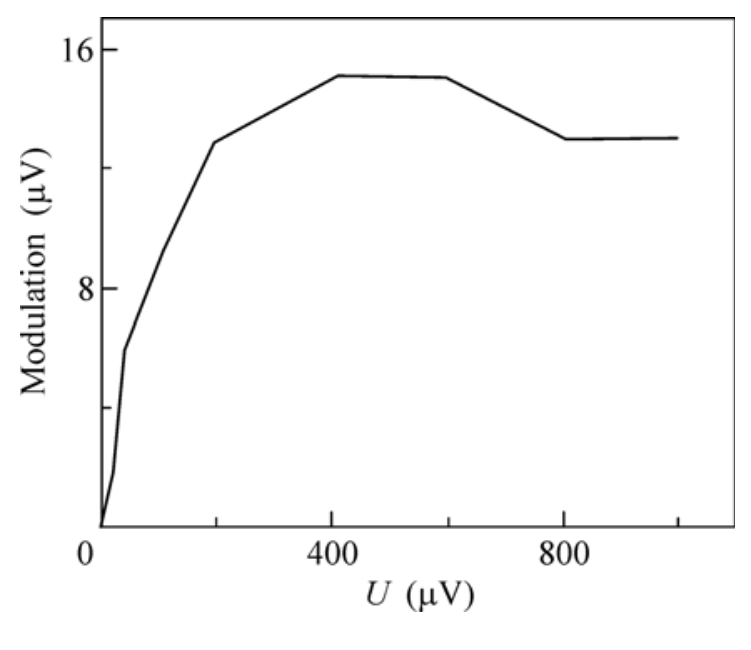

Fig. 6. Modulation depth versus the bias voltage on the interferometer.

no. 11-02-12145-ofi-m-2011. The samples were manufactured in a clean room with equipment subsidized by the Knut and Alice Wallenberg Foundation.

\section{REFERENCES}

1. X. Du, I. Skachko, and E. Y. Andrei, Phys. Rev. B 77, 184507 (2008).

2. F. Miao, C. N. Lau, et al., Science 317, 1530 (2007).

3. F. Morpurgo et al., Nature 446, 56 (2007).

4. C. Girit, I. Siddiqi, et al., Nano Lett. 9, 198 (2009).

5. L. Kuzmin, Proc. SPIE 5498, 349 (2004).

6. L. Kuzmin and D. Golubev, Physica C 372-376, 378 (2002).

7. A. Agulo, L. Kuzmin, and M. Tarasov, in Proceedings of the 16th ISSTT (2005), p. 147.

8. M. Tarasov, L. Kuzmin, N. Kaurova, et al., in Proceedings of the 21st ISSTT (2010).

9. M. Tarasov, J. Svensson, L. Kuzmin, and E. Campbell, Appl. Phys. Lett. 90, 163503 (2007).

Translated by R. Tyapaev 